\title{
Comparación de la influencia de las pérdidas energéticas en las eficiencias de un compresor de desplazamiento (scroll) y un compresor de pistones utilizados en bombas de calor
}

\author{
Fernando M. Tello-Oquendo', Daniela C. Vásconez-Núñez ${ }^{1}$ y Emilio Navarro-Peris ${ }^{2}$ \\ (1) Grupo de Investigación y Desarrollo en Nanotecnología, Materiales y Manufactura (GIDENM), Facultad de Mecánica, \\ Escuela Superior Politécnica de Chimborazo, Panamericana Sur km 1 11/2, Riobamba, Ecuador. \\ (correo-e: fernando.tello@espoch.edu.ec; daniela.vasconez@espoch.edu.ec) \\ (2) Instituto Universitario de Investigación en Ingeniería Energética (IUIIE), Universitat Politècnica de València, Valencia, \\ 46022, España. (correo-e: emilio.navarro@iie.upv.es)
}

Recibido Abr. 14, 2020; Aceptado Jun. 10, 2020; Versión final Jul. 15, 2020, Publicado Oct. 2020

\begin{abstract}
Resumen
Este artículo presenta la comparación de la influencia de las pérdidas energéticas en las eficiencias de un compresor de desplazamiento scroll y un compresor de pistones. Las influencias de las pérdidas del compresor scroll se estimaron con un modelo semi-empírico validado experimentalmente. Para el compresor de pistones se utilizaron datos de la literatura previa. Respecto a la eficiencia isentrópica, las pérdidas mecánicas, eléctricas y las fugas presentan una influencia similar en los dos compresores de aproximadamente el $90 \%$; la transferencia de calor y las pérdidas de presión en la succión son $7 \%$ más influyentes en el compresor de pistones. Respecto a la eficiencia volumétrica, las pérdidas mecánicas, eléctricas y las fugas son aproximadamente 39\% más influyentes en el compresor scroll y la influencia de las pérdidas de presión en la succión y la transferencia de calor son $21 \%$ mayores en el compresor de pistones. Se concluye que el compresor de pistones tiene un mayor flujo másico de fugas.
\end{abstract}

Palabras clave: compresor scroll; compresor de pistones; pérdidas energéticas; eficiencia

\section{Comparison of the influence of energy losses on efficiencies of a scroll compressor and a reciprocating compressor used in heat pumps}

\begin{abstract}
This paper presents the comparison of the influence of energy losses on the efficiencies of a scroll compressor and a reciprocating compressor. The influences of the scroll compressor losses are estimated with an experimentally validated semi-empirical model. For the reciprocating compressor, data from the previous literature is used. Regarding isentropic efficiency, mechanical, electrical losses, and leaks have a similar influence on the two compressors of approximately $90 \%$. Heat transfer and suction pressure losses are $7 \%$ more influential in the reciprocating compressor. Regarding volumetric efficiency, mechanical, electrical losses, and leaks are approximately $39 \%$ more influential in the scroll compressor, and the influence of suction pressure losses and heat transfer are $21 \%$ greater in the reciprocating compressor. It is concluded that the reciprocating compressor has a higher leaked mass flow rate.
\end{abstract}

Keywords: scroll compressor; reciprocating compressor; energy losses; efficiency 


\section{INTRODUCCIÓN}

Hoy en día, los compresores scroll son ampliamente utilizados en aplicaciones residenciales y comerciales de aire acondicionado, de refrigeración y bombas de calor, así como en aire acondicionado automotriz. Esta tecnología de compresor es de movimiento orbital, son máquinas de desplazamiento positivo que comprimen con dos elementos de desplazamiento en forma de espiral que se ajustan entre sí. Dichos elementos espirales no tienen espacio muerto, el contacto entre los flancos de los espirales y en sus bases y bordes superiores es casi perfecto y constante; por lo tanto, tiene buena conformidad axial y radial. En consecuencia, los compresores scroll presentan varias ventajas, como una alta eficiencia volumétrica e isentrópica, bajas vibraciones y ruido, bajas variaciones de par y fugas (ASHRAE Handbook, 2016).

Otra importante tecnología de compresores utilizada en sistemas de refrigeración y bombas de calor son los compresores de pistones. Estos compresores presentan unas curvas de eficiencia isentrópica más planas que la de los compresores scroll, lo que permite mantener la eficiencia isentrópica en un amplio rango de relaciones de presiones. No así, la eficiencia volumétrica que decae rápidamente cuando la relación de presiones incrementa. En este contexto, para determinar la tecnología adecuada para cada aplicación es necesario analizar tanto las eficiencias isentrópica y volumétrica, como las pérdidas energéticas de las tecnologías de compresión y su influencia en dichas eficiencias. Una metodología para el estudio de las pérdidas energéticas se basa en la utilización de modelos de compresores, debido a la dificultad de evaluar dichas pérdidas de manera experimental.

En la literatura se han encontraron varios modelos de compresores scroll que se han utilizado para estimar las eficiencias y para evaluar el COP de una bomba de calor o una máquina de refrigeración en diversas condiciones de funcionamiento. Winandy et al. (2002), Cuevas y Lebrun (2009), y Cuevas et al. (2010) trabajaron en un modelo de compresor scroll simplificado basado en los procesos principales que afectan al refrigerante durante la compresión. El modelo utiliza una pared isotérmica ficticia para modelar los intercambios de calor dentro del compresor y entre el compresor y el ambiente. El modelo calcula el caudal másico, la potencia eléctrica del compresor y la temperatura de descarga del refrigerante con desviaciones inferiores a $\pm 10 \%, \pm 10 \%$ y $\pm 5 \mathrm{~K}$, respectivamente, en condiciones típicas de funcionamiento. A partir del modelo de Winandy et al. (2002), se han derivado varios trabajos sobre compresores scroll de simple etapa (Duprez et al., 2007 y 2010), compresores que trabajan con hidrocarburos (Byrne et al., 2014), compresores scroll inundados de aceite con inyección de líquido (James et al., 2016) y compresores scroll de velocidad variable con inyección de vapor (Dardenne et al., 2015). En todos estos estudios, se consideran algunas fuentes de pérdidas energéticas como las transferencias térmicas en la succión por calentamiento y en la descarga por enfriamiento, fugas internas, pérdidas mecánicas, eléctricas y pérdidas al ambiente. Sin embargo, estos estudios no presentan una estimación de la influencia de las fuentes de pérdidas energéticas en las eficiencias isentrópica y volumétrica del compresor scroll.

Por otro lado, Bell et al. (2013) propusieron un método para obtener correlaciones para el cálculo de fugas radiales y tangenciales en compresores scroll. Su método se basó en el desarrollo de una correlación empírica para el modelo de tobera isentrópica utilizando un gran conjunto de datos numéricos obtenidos del modelo detallado unidimensional de la ruta del flujo de fuga dentro del compresor. Pereira y Deschamps (2020) analizaron numéricamente las fugas radiales y tangenciales de un compresor scroll, considerando diferentes refrigerantes, condiciones de operación y geometrías de las espirales del compresor. Sin embargo, en estos estudios no se estimó el peso ponderado de las fugas internas del compresor scroll en las eficiencias isentrópica y volumétrica del compresor.

Tello-Oquendo et al. (2018) presentaron un modelo semi-empírico de un compresor scroll y su extensión para describir compresores scroll con inyección de vapor. El modelo toma en cuenta la evolución ideal del refrigerante en el compresor y considera las principales fuentes de pérdidas en el proceso de compresión. El modelo reproduce la eficiencia isentrópica y la eficiencia volumétrica con una desviación inferior a $\pm 5 \%$ y $\pm 3 \%$, respectivamente, en un amplio rango de condiciones de operación. Además, el modelo estima el caudal másico, la potencia eléctrica del compresor y la temperatura de descarga con una desviación inferior a $\pm 3 \%$, $\pm 5 \%$ y $\pm 3 \mathrm{~K}$, respectivamente. Con este modelo los autores realizaron una estimación de las pérdidas en el proceso de compresión, considerando las limitaciones intrínsecas que presentan los modelos semi-empíricos.

Con respecto a los compresores de pistones, Arencibia y Tricio (2014) estudiaron experimentalmente la capacidad frigorífica y la eficiencia de tres compresores herméticos de pistones de una sola etapa bajo condiciones de clima tropical. Los resultados muestran que bajo condiciones de temperatura ambientales entre $32-35^{\circ} \mathrm{C}$, la capacidad frigorífica disminuye $10 \%$ y la eficiencia isentrópica $13 \%$ comparando con el rendimiento del sistema bajo condiciones ASHRAE CECOMAF. Roskosch et al. (2017) utilizaron un modelo de compresor de pistones para calcular las eficiencias isentrópica y volumétrica en función de las condiciones de succión, presión de descarga y el refrigerante. El estudio consideró como parámetro el volumen muerto, la presión de fricción y las áreas de flujo efectivas en las válvulas de succión y descarga. Tuhovcak et al. 
(2016) analizaron el efecto de la transferencia de calor dentro del pistón sobre la eficiencia isentrópica del compresor utilizando varios refrigerantes. Navarro et al. (2007a, 2007b) presentaron dos trabajos sobre un modelo semi-empírico de compresores de pistones que calcula la eficiencia isentrópica y la eficiencia volumétrica en términos de diez parámetros que representan las principales fuentes de pérdidas dentro del compresor. El modelo reproduce el funcionamiento del compresor y las eficiencias volumétricas con un error inferior al 3\% en una amplia gama de condiciones de operación. Además, se analizó la influencia relativa de las pérdidas en la eficiencia isentrópica en términos de las condiciones de funcionamiento. Los resultados del modelo muestran que las pérdidas mecánicas y eléctricas representan aproximadamente el $75 \%$ de las pérdidas de eficiencia isentrópica y el $55 \%$ de las pérdidas de eficiencia volumétrica. A relaciones de presiones bajas (1.5-2.5), las pérdidas de presión son notables (más del 15\% de influencia en la eficiencia isentrópica y más del $25 \%$ de influencia en la eficiencia volumétrica). A relaciones de presiones altas (5-7), las fugas se convierten en un factor significativo (más del 10\% de influencia en las eficiencias isentrópica y volumétrica). La influencia relativa de las pérdidas de transferencia de calor entre la succión y la descarga permanece constante en todas las relaciones de presión y su influencia general sobre la eficiencia volumétrica es significativa (15\%).

La revisión bibliográfica contempla importantes estudios sobre las eficiencias de los compresores scroll y de pistones que consideran las principales fuentes de pérdidas energéticas, no obstante, no se han encontrado análisis comparativos entre las pérdidas de las dos tecnologías de compresor en donde se contrasten las influencias de cada fuente de pérdida en la reducción de las eficiencias de los compresores.

El presente artículo aborda la comparación de la influencia relativa de las principales fuentes de pérdidas en las eficiencias de un compresor scroll y un compresor de pistones. Las influencias relativas de las pérdidas en las eficiencias de los compresores se estiman en función de las condiciones de trabajo (relación de presiones). Para este propósito, se implementa un modelo semi-empírico de un compresor scroll de acuerdo a la referencia Tello-Oquendo et al. (2018). La validación del modelo se realiza utilizando datos experimentales de un compresor scroll, que se ensaya en un banco de pruebas calorimétricas en una amplia gama de condiciones de funcionamiento. La comparación de las influencias relativas de las pérdidas en las eficiencias de los compresores se realiza para una condición de trabajo nominal, usando propano como refrigerante. Los datos correspondientes al compresor de pistones se obtuvieron de la referencia Navarro et al. (2007a).

\section{METODOLOGÍA}

La instalación experimental utilizada para ensayar el compresor scroll consiste en un banco de pruebas calorimétrico. La Figura 1 muestra el esquema del banco de ensayos utilizado, el cual fue diseñado para controlar las condiciones de operación en los puertos de succión y descarga del compresor (puntos (1) y (8) en la Figura 1). El compresor utilizado para la caracterización fue un compresor scroll de $9.95 \mathrm{~m}^{3} \cdot \mathrm{h}^{-1}$ de volumen desplazado. El compresor se ensayó con R-290 como refrigerante y se utilizó aceite POE ISO 32.

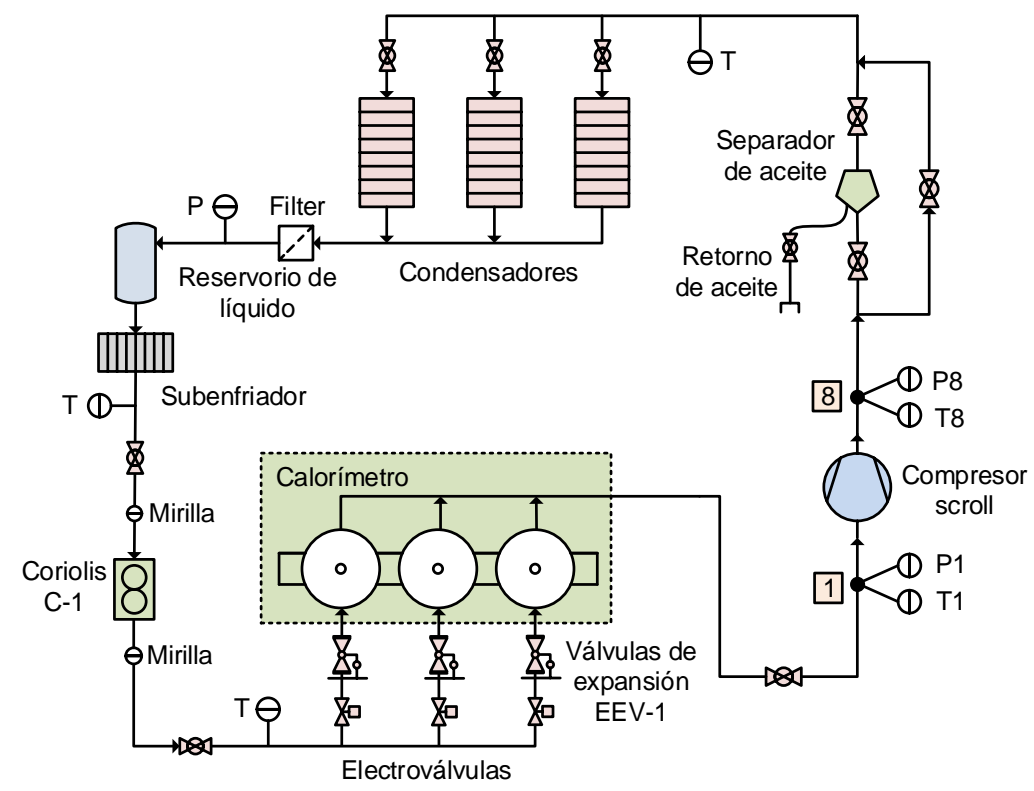

Fig. 1: Esquema del banco calorimétrico. 
El procedimiento de ensayo del compresor se realizó de acuerdo con la Norma EN 13771-1 (2017). El caudal másico del refrigerante es el parámetro determinante a medir. El procedimiento de prueba primario elegido es el método del calorímetro de refrigerante secundario y como método de confirmación se utilizó un medidor de flujo másico de tipo Coriolis. El caudal másico del evaporador se midió directamente utilizando un medidor tipo Coriolis (C-1 en la Figura 1). Este caudal másico medido se comparó con el resultado del calorímetro secundario basado en el refrigerante. En base a la norma EN 13771-1 (2017), los ensayos son válidos si las desviaciones entre el método primario y el secundario de medición del caudal másico es inferior al $4 \%$. Sin embargo, se obtuvo desviaciones inferiores al $2 \%$ en el banco de pruebas calorimétrico para todos los puntos ensayados.

Se incorporaron varios lazos de control PID para permitir un ajuste preciso de las condiciones del refrigerante en la entrada del compresor (temperatura de evaporación $\left(\mathrm{T}_{e}\right)$ y sobrecalentamiento $(\mathrm{SH})$ ) y la salida (temperatura de condensación $\left(T_{c}\right)$ ). El banco calorimétrico está completamente automatizado y diseñado para alcanzar cualquier condición de prueba permitida sin ajustes manuales. Las precisiones de los instrumentos se detallan en la Tabla 1. La seguridad fue una preocupación importante durante el diseño de las instalaciones de prueba. Se tomaron en cuenta los procedimientos y estándares específicos con respecto al manejo y uso de gases inflamables. Las medidas específicas incluyeron el uso de material eléctrico intrínsecamente seguro, sensores de propano específicos, el uso de interruptores y alarmas de emergencia y procedimientos de renovación de aire apropiados para garantizar concentraciones no críticas en el caso de fugas (EN 378, 2017).

Tabla 1: Cantidad y precisión de los instrumentos de medición

\begin{tabular}{|c|l|l|}
\hline Cantidad & \multicolumn{1}{|c|}{ Instrumento } & \multicolumn{1}{|c|}{ Precisión } \\
\hline 1 & Coriolis Fisher-Rosemount Micro-Motion CMF025M & $\pm 0.025 \mathrm{~g} \cdot \mathrm{s}^{-1}$ \\
\hline 3 & Transductor de presión Fisher-Rosemount 3051 & $\pm 1.00 \mathrm{kPa}$ \\
\hline 5 & Transmisor de temperatura RTD-PT 100 & $\pm 0.05 \mathrm{~K}$ \\
\hline 1 & Medidor de potencia eléctrica & $0.10 \%$ \\
\hline
\end{tabular}

La Tabla 2 muestra la matriz de ensayos realizados para caracterizar el compresor scroll. En la Tabla, "a" es el punto de ensayo para la caracterización del compresor, y "b" es el punto de ensayo usado en la comparación de las pérdidas de un compresor scroll y un compresor de pistones. El procedimiento de caracterización del compresor comienza con el ajuste de la presión de condensación, la presión de evaporación y el recalentamiento en la entrada del compresor actuando sobre el caudal de agua del condensador, las válvulas EEV-1 y las resistencias del calorímetro, respectivamente. Para todos los puntos de operación $\left(T_{e}, T_{c}\right)$, un sobrecalentamiento en la entrada del compresor de $10 \mathrm{~K}$ fue considerado como un parámetro en la caracterización del compresor. Una vez que el sistema está en equilibrio, se miden el caudal másico del evaporador $\left(\dot{m}_{i n}\right)$ y la potencia eléctrica del compresor. Además, se registra la temperatura de descarga $\left(T_{8}\right)$.

Tabla 2: Matriz de ensayos del compresor scroll

\begin{tabular}{|c|l|l|l|l|l|l|l|l|l|l|}
\hline \multirow{2}{*}{$\begin{array}{l}\text { Temperatura de } \\
\text { condensación }\left({ }^{\circ} \mathrm{C}\right)\end{array}$} & \multicolumn{7}{|c|}{ Temperatura de evaporación $\left({ }^{\circ} \mathrm{C}\right)$} \\
\hline & -30 & -14 & -7 & -5 & 0 & 5 & 7 & 10 & 20 \\
\hline 35 & $\mathrm{a}$ & & $\mathrm{a}$ & & & & & & \\
\hline 45 & $\mathrm{a}$ & & & & & & & & $\mathrm{a}$ \\
\hline 50 & $\mathrm{a}$ & & $\mathrm{a}, \mathrm{b}$ & & & $\mathrm{a}$ & & & \\
\hline 54 & & & & & & & $\mathrm{a}$ & & \\
\hline 60 & & & & & $\mathrm{a}$ & & & & $\mathrm{a}$ \\
\hline 70 & & $\mathrm{a}$ & & $\mathrm{a}$ & & & & $\mathrm{a}$ & \\
\hline
\end{tabular}

La eficiencia volumétrica está definida por la ecuación (1), donde el número 1 se encuentra en la entrada del compresor. La eficiencia isentrópica del compresor está definida por la ecuación (2). Esta expresión representa la relación entre la potencia del proceso isentrópico y la potencia eléctrica suministrada al compresor.

$$
\begin{aligned}
& \eta_{\mathrm{v}}=\frac{\dot{\mathrm{m}}_{\text {in }}}{\rho_{1} \dot{\mathrm{V}}_{\mathrm{s}}} \\
& \eta_{\mathrm{c}}=\frac{\dot{\mathrm{m}}_{\mathrm{in}}\left(\mathrm{h}_{8 \mathrm{~s}}-\mathrm{h}_{1}\right)}{\dot{\mathrm{E}}}
\end{aligned}
$$


En estas ecuaciones, $h_{8 s}$ representa la entalpía específica en $(\mathrm{kJ} / \mathrm{kg})$ a la presión de descarga del compresor, considerando una compresión isentrópica desde la condición de entrada del compresor (ver punto 1 en la Figura 1) y Ė representa la potencia eléctrica del compresor en (kW). Las temperaturas de evaporación y condensación son temperaturas de vapor saturado. Las propiedades termofísicas del refrigerante en los diferentes puntos se calculan con la base de datos NIST REFPROP (Lemmon et al., 2010).

El modelo del compresor que se implementó se basa en el modelo semi-empírico según Tello-Oquendo et al. (2018). Dicho modelo reproduce la eficiencia isentrópica del compresor y la eficiencia volumétrica en función de un conjunto de parámetros que pueden obtenerse mediante correlaciones de los datos de caracterización estándar del compresor. Estos parámetros tienen un trasfondo físico para que, una vez correlacionados, el modelo se pueda usar para predecir el rendimiento del compresor en condiciones de funcionamiento que no se han ensayado. La Figura 2a representa la evolución del refrigerante en un diagrama P-h asumido por el modelo. El refrigerante ingresa al compresor en el punto 1. La referencia para la eficiencia isentrópica del compresor viene dada por una condición isentrópica desde la entrada a la salida del compresor (8s). Las condiciones reales en la salida del compresor se indican mediante el estado 8 en la Figura 2a. El modelo asume que la evolución del refrigerante a través del compresor se divide en la siguiente secuencia de efectos: (1-2): calentamiento isobárico de vapor debido al enfriamiento del motor y la disipación de pérdidas mecánicas. (2-3): calentamiento isobárico de vapor debido al calor transferido desde el lado caliente del compresor (descarga) al flujo de entrada y a las fugas. (3-4): pérdida de presión isoentálpica en el puerto de succión. (4-ad): compresión isentrópica desde las condiciones de admisión de los scrolls (aparecen fugas en esta parte del proceso) hasta la presión adaptada en el puerto de descarga. (ad-5): compresión isocórica desde la presión adaptada a la presión de descarga $\left(P_{c}\right)$ en la cámara de descarga. (5-6): pérdida de presión isoentálpica en el puerto de descarga. (6-7): enfriamiento isobárico de vapor debido al calor transferido al lado de succión. (7-8): pérdida de calor al ambiente.

a)

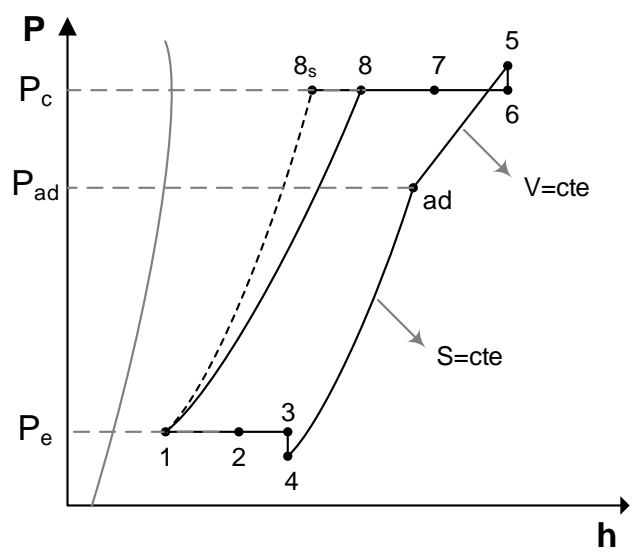

b)

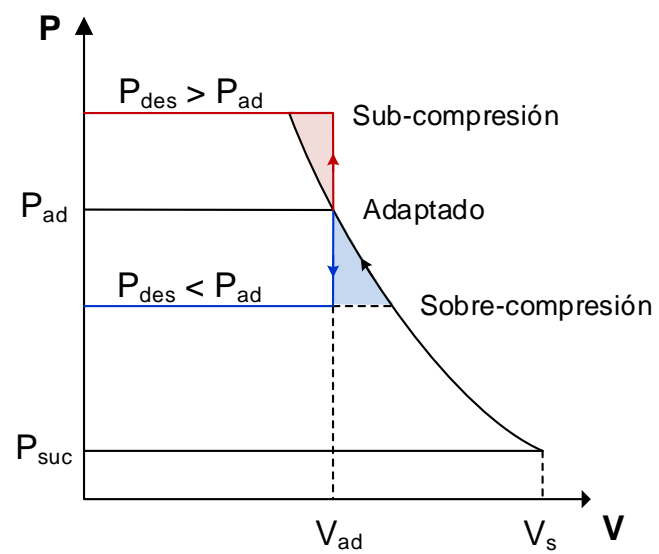

Fig. 2: a) Evolución del refrigerante dentro del compresor scroll (sub-compresión). b) Diagrama P-V del proceso de compresión del compresor scroll (Adaptada de Tello-Oquendo et al., 2018).

Con respecto al proceso de 4 a 5 , la compresión en compresores scroll incorpora una relación fija de volumen $(\varepsilon)$, que se define por la geometría de los scrolls y por la ubicación del puerto de descarga (ecuación (3)). La relación de volumen $(\varepsilon)$ no se conoce a priori y debe identificarse. La variable conocida es el volumen desplazado en la succión $\left(\dot{\mathrm{V}}_{\mathrm{s}}\right)$, que es proporcionado por el fabricante del compresor. Para un refrigerante dado y determinadas condiciones de funcionamiento, existe una relación de presión interna fija que corresponde a la relación de volumen incorporada. Por lo tanto, si la relación de presión externa (definida por las condiciones de trabajo de una aplicación dada) es diferente a la relación de presión interna, el compresor no está adaptado. En este contexto, se distinguen tres situaciones posibles: cuando la relación de presión externa $\left(P_{r}\right)$ es igual a la relación de presión interna (adaptada), cuando $P_{r}$ es mayor a la relación de presión interna (subcompresión) y cuando $P_{r}$ es menor a la relación de presión interna (sobrecompresión). Estas tres situaciones posibles se ilustran en el diagrama P-V de la Figura 2b. Cuando el compresor no está adaptado, se generan pérdidas de potencia en relación con el proceso adaptado (Winandy et al., 2002). Las principales ecuaciones del modelo del compresor scroll se resumen a continuación (Tello-Oquendo et al., 2018):

$$
\begin{aligned}
& \varepsilon=\frac{\dot{\mathrm{V}}_{\mathrm{s}}}{\dot{\mathrm{V}}_{\mathrm{ad}}} \\
& \Delta \mathrm{T}_{1-2}=\frac{\dot{\mathrm{Q}}_{1-2}}{\dot{\mathrm{m}}_{\mathrm{in}} \mathrm{C}_{\mathrm{p} 1}}=\mathrm{K}_{1}\left(\frac{\left(1-\eta_{\mathrm{el}}\right)}{\eta_{\mathrm{c}}} \frac{\mathrm{h}_{8 \mathrm{~s}}-\mathrm{h}_{1}}{\mathrm{C}_{\mathrm{p} 1}}+\frac{\dot{\mathrm{E}}_{\mathrm{mec}}}{\eta_{\mathrm{v}} \dot{\mathrm{V}}_{\mathrm{s}} \rho_{1} \mathrm{C}_{\mathrm{p} 1}}\right)
\end{aligned}
$$




$$
\begin{aligned}
& \Delta \mathrm{T}_{2-3}=\frac{\dot{\mathrm{Q}}_{2-3}}{\dot{\mathrm{m}}_{\mathrm{in}} \mathrm{C}_{\mathrm{p} 2}}=\mathrm{K}_{2}\left(\frac{\mathrm{T}_{8 \mathrm{~s}}-\mathrm{T}_{1}}{\eta_{\mathrm{v}} \dot{\mathrm{V}}_{\mathrm{s}} \rho_{1} \mathrm{C}_{\mathrm{p} 2}}\right) \\
& \Delta \mathrm{P}_{3-4}=\mathrm{K}_{3} \rho_{3}\left(\mathrm{\eta}_{\mathrm{v}} \dot{\mathrm{V}}_{\mathrm{s}}\right)^{2} \\
& \Delta \mathrm{P}_{5-6}=\mathrm{K}_{4} \rho_{5}\left(\frac{\rho_{4}}{\rho_{5}} \eta_{v} \dot{\mathrm{V}}_{\mathrm{s}}\right)^{2} \\
& \Delta \mathrm{T}_{6-7}=\frac{\dot{\mathrm{Q}}_{2-3}}{\dot{\mathrm{m}}_{\mathrm{in}} \mathrm{C}_{\mathrm{p} 6}} \\
& \Delta \mathrm{T}_{7-8}=\frac{\dot{\mathrm{Q}}_{\mathrm{amb}}}{\dot{\mathrm{m}}_{\mathrm{in}} \mathrm{C}_{\mathrm{p} 7}}=U \mathrm{~A}_{\mathrm{amb}} \frac{\mathrm{T}_{7}-\mathrm{T}_{\mathrm{amb}}}{\dot{\mathrm{m}}_{\mathrm{in}} \mathrm{C}_{\mathrm{p} 7}} \\
& \dot{\mathrm{E}} \mathrm{L}_{\mathrm{mec}}=\mathrm{K}_{5} \dot{\mathrm{E}}+\mathrm{K}_{6} \mathrm{n}^{2} \\
& \left.\dot{\mathrm{m}}_{\text {fugas }}=A_{\text {fugas }} \rho_{\left(P_{g},\right. \text { fugas }}, s_{5}\right) \sqrt{2\left(h_{5}-h_{\left(P_{g, f u g a s}\right.}, s_{5}\right)} \\
& \dot{\mathrm{m}}_{\mathrm{e}} \mathrm{h}_{4}+\dot{\mathrm{m}}_{\text {fugas }} \mathrm{h}_{5}=\left(\dot{\mathrm{m}}_{\mathrm{e}}+\dot{\mathrm{m}}_{\text {fugas }}\right) \mathrm{h}_{4^{\prime}} \\
& \dot{\mathrm{E}}=\frac{1}{\eta_{\mathrm{el}}}\left(\dot{\mathrm{W}}_{4^{\prime}-5}+\dot{\mathrm{E}}_{\mathrm{mec}}\right) \\
& \dot{\mathrm{W}}_{4-5}=\left(\dot{\mathrm{m}}_{\text {in }}+\dot{\mathrm{m}}_{\text {fugas }}\right)\left(\mathrm{h}_{\mathrm{ad}}-\mathrm{h}_{4^{\prime}}\right)+\dot{\mathrm{V}}_{\mathrm{ad}}\left(\mathrm{P}_{5^{-}}-\mathrm{P}_{\mathrm{ad}}\right)
\end{aligned}
$$

Las ecuaciones de la (4) a la (9) estiman las variaciones de la temperatura y presión del refrigerante dentro del compresor en la succión y descarga (ver evolución del refrigerante en la Figura 2a). Las pérdidas mecánicas del compresor scroll se estiman con la ecuación (10). Esta ecuación considera la suma de dos términos, uno proporcional a la potencia eléctrica del compresor y el otro dependiente de la velocidad del compresor. Todas las fugas internas (laterales y radiales) que ocurren continuamente en el compresor a medida que avanza la compresión se modelan como un flujo isentrópico del gas compresible a través de una tobera convergente simple (ecuación (11)), según Giuffrida (2014). El parámetro para el cálculo del flujo másico de fugas ( $\dot{\mathrm{m}}_{\text {fugas }}$ ) es Afugas, que representa el área de la sección transversal de la garganta de la tobera. La presión de la garganta $\left(\mathrm{P}_{\mathrm{g}}\right.$,ugas $)$ es el máximo valor entre la presión real de la garganta de la tobera $\mathrm{P}_{4}$ y la presión crítica. El modelo supone una mezcla entre el flujo de refrigerante fugado con el flujo de refrigerante en la succión de manera isobárica y adiabática según la ecuación (12). La potencia eléctrica del compresor

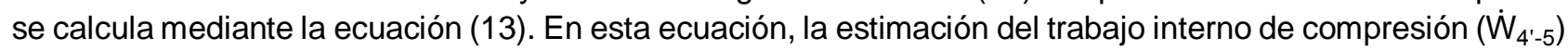
se lo realiza con la ecuación (14). Para formular el modelo global, las ecuaciones que rigen las diferentes pérdidas se introducen en las ecuaciones (1) y (2) por sustitución directa de las ecuaciones obtenidas (ecuaciones (10), (13), y (14)), y también se utilizan para calcular los estados del refrigerante 4, 5, 6, 7 y 8 (ecuaciones (4) - (9)), que conducen a un sistema de dos ecuaciones implícitas para las eficiencias isentrópica y volumétrica (ecuaciones (15) y (16)).

$$
\begin{aligned}
& f_{1}\left(\eta_{c}, \eta_{v}, K_{i}, A_{\text {fugas }}, U A_{a m b}, \eta_{e l}, \varepsilon, \dot{V}_{s}, P_{1}, P_{8}, S H\right)=0 \\
& f_{2}\left(\eta_{c}, \eta_{v}, K_{i}, A_{\text {fugas }}, U A_{a m b}, \eta_{e l}, \varepsilon, \dot{V}_{s}, P_{1}, P_{8}, S H\right)=0
\end{aligned}
$$

donde $K_{i}=\left(K_{1}, \ldots, K_{6}\right)$, representan los parámetros de diseño del compresor. Una vez que se conocen todos los parámetros de diseño del compresor, se puede resolver el sistema de dos ecuaciones para las eficiencias isentrópica y volumétrica, para una condición de trabajo dada $\left(\mathrm{P}_{1}, \mathrm{P}_{8}, \mathrm{SH}\right)$. Se puede emplear cualquier motor de cálculo para un sistema de ecuaciones no lineales. Se requiere un conjunto de datos para una serie de condiciones de trabajo obtenidas de experimentos o de catálogos de fabricantes para obtener el valor adecuado de $\mathrm{K}_{\mathrm{i}}$, mediante un procedimiento de ajuste. Los resultados que se muestran en este trabajo se obtuvieron mediante el método de gradiente conjugado multidimensional (Press et al., 2007). El modelo del compresor se implementó en el software EES (Klein y Alvarado, 2017).

Cada parámetro del modelo está asociado a una pérdida de energía específica. Las pérdidas de energía consideradas en el modelo incluyen: i) las pérdidas mecánicas $\left(K_{1}, K_{5}\right.$ y $\left.K_{6}\right)$, ii) pérdidas eléctricas $\left(\eta_{e l}\right)$, iii) caídas de presión en la succión ( $\left.\mathrm{K}_{3}\right)$, iv) caídas de presión en la descarga $\left(\mathrm{K}_{4}\right)$, v) pérdidas por fugas (Afugas), vi) pérdidas por transferencia de calor entre la succión y descarga $\left(K_{2}\right)$. Para analizar las pérdidas de energía del compresor se estima la influencia relativa de cada pérdida en las eficiencias del compresor, utilizando la 
ecuación (17). La influencia relativa representa el peso ponderado de cada pérdida en la reducción de las eficiencias isentrópica y volumétrica del compresor.

Influencia relativa de la pérdida, $i(\%)=\frac{1-\eta_{(\text {pérdida }, i=0)}}{1-\eta} * 100$

\section{RESULTADOS Y DISCUSIÓN}

Los resultados del presente estudio se muestran en dos subsecciones: en la primera se describe la validación del modelo semi-empírico utilizado para el compresor scroll; $y$ en la segunda se compara la influencia relativa de las pérdidas energéticas sobre las eficiencias del compresor scroll y de un compresor de pistones.

\section{Validación del modelo del compresor scroll}

La Figura 3 ilustra las eficiencias volumétrica e isentrópica del compresor en función de la relación de presiones. Esta figura muestra una eficiencia isentrópica máxima para una relación de presión de alrededor de 3, que corresponde con la condición de adaptación del compresor, donde la relación de presión externa $\left(P_{r}\right)$ es igual a la relación de presión interna. Este valor puede usarse como una primera aproximación de $(\varepsilon)$ en el proceso de ajuste, considerando que una relación de presiones dada corresponde a la misma relación de volúmenes del compresor.

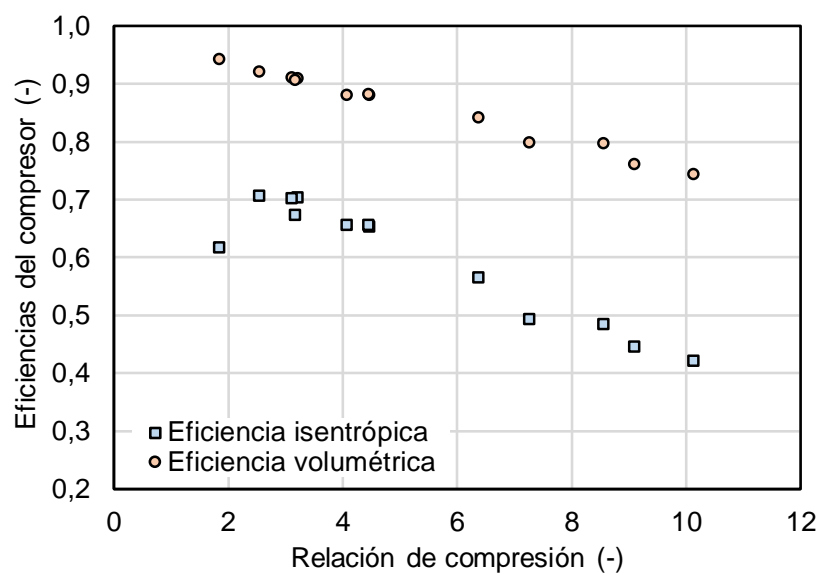

Fig. 3: Eficiencias volumétrica e isentrópica experimentales del compresor scroll.

Utilizando los datos experimentales de la caracterización del compresor, se ajustó el modelo descrito anteriormente. Los parámetros del modelo del compresor scroll encontrados en el proceso de ajuste son: $\mathrm{K}_{1}$ $=1.5, \mathrm{~K}_{2}=0.15 ; \mathrm{K}_{3}=1 \times 104 ; \mathrm{K}_{4}=1.64 \times 10^{9} ; \mathrm{K}_{5}=52 ; \mathrm{K}_{6}=118 ; \varepsilon=2.99 ; \mathrm{A}_{\text {leak }}=8.1 \times 10^{-6} \mathrm{~m}^{2} ; \mathrm{UA}_{\mathrm{amb}}=0.0045$ $\mathrm{kW} \mathrm{K} \mathrm{K}^{-1} ; \eta_{\mathrm{el}}=0.89$. Las figuras $4 \mathrm{a}$ y $4 \mathrm{~b}$ muestran la comparación entre los resultados experimentales y los calculados de las eficiencias del compresor utilizando el modelo. Los resultados muestran un correcto ajuste entre los parámetros experimentales y calculados con una desviación inferior al $\pm 5 \%$.

a)

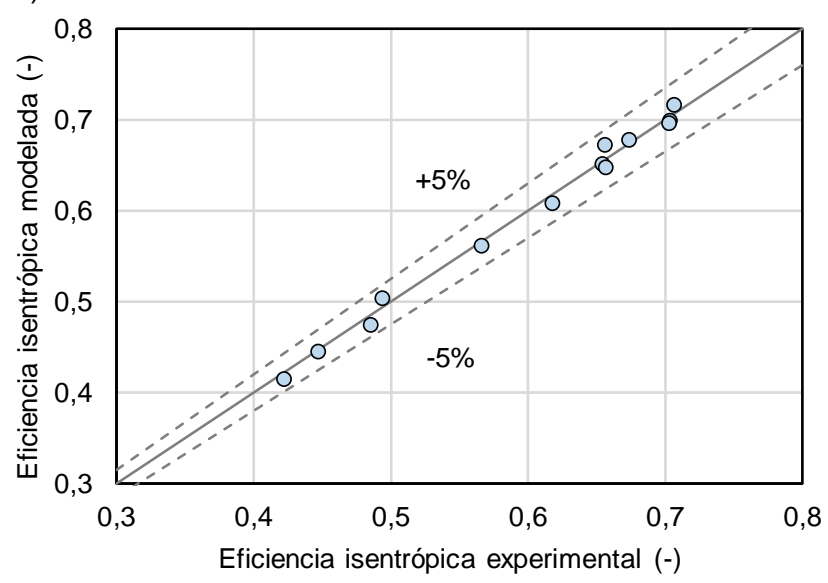

b)

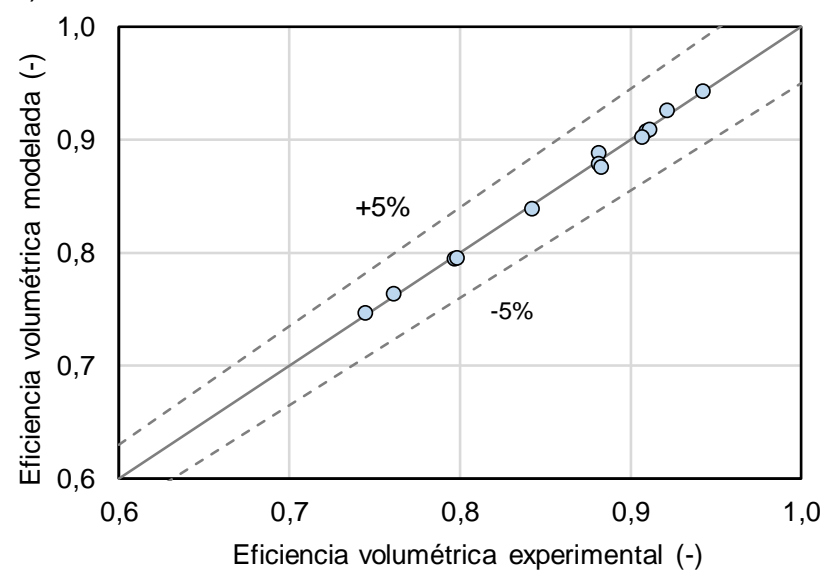

Fig. 4: a) Estimación de la eficiencia isentrópica del compresor scroll. b) Estimación de eficiencia volumétrica del compresor scroll. 


\section{Comparación de influencia relativa de las pérdidas sobre las eficiencias volumétrica e isentrópica}

La Figura 5 ilustra la influencia relativa de las pérdidas en las eficiencias volumétrica e isentrópica del compresor scroll, para todas las condiciones de ensayo disponibles en función de la relación de presiones. Debe tomarse en cuenta que los valores calculados no representan valores absolutos de pérdidas. La Figura 5a muestra que las pérdidas eléctricas y mecánicas son las más influyentes en la reducción de la eficiencia isentrópica del compresor (aproximadamente un 82\%), las pérdidas de presión presentan poca influencia en la eficiencia isentrópica del compresor. Las fugas alcanzan hasta un $12 \%$ de influencia relativa en la reducción de la eficiencia isentrópica del compresor para las relaciones de presiones más altas (8-10). La transferencia de calor entre las cámaras de succión y descarga es responsable de aproximadamente el $4 \%$ de la reducción en la eficiencia isentrópica. Con respecto a la eficiencia volumétrica de la Figura $5 b$, se puede concluir que las pérdidas eléctricas y mecánicas también son considerables, y su influencia permanece aproximadamente constante en todas las condiciones de trabajo (aproximadamente el $70 \%$ ). Las pérdidas de presión tienen poca influencia y las fugas presentan una influencia de hasta el $28 \%$. La influencia de la transferencia de calor entre las cámaras de succión y descarga es menor al $8 \%$.

a)

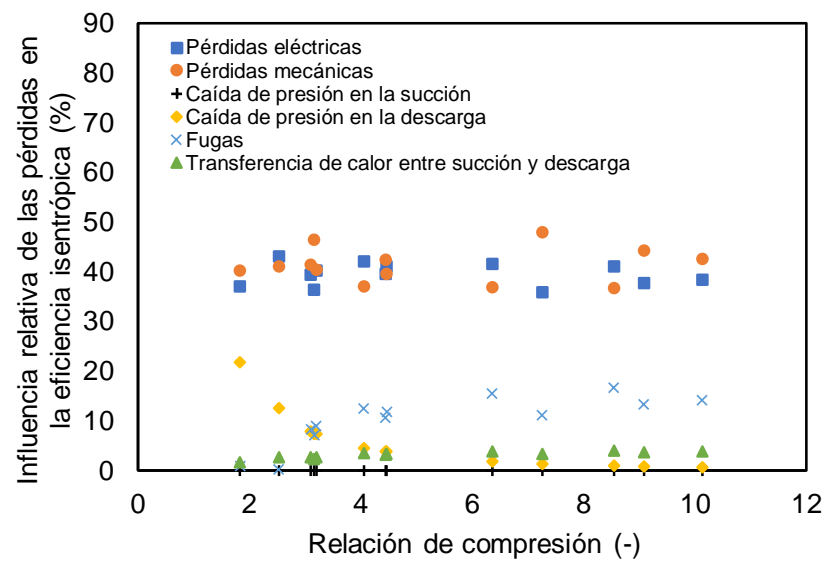

b)

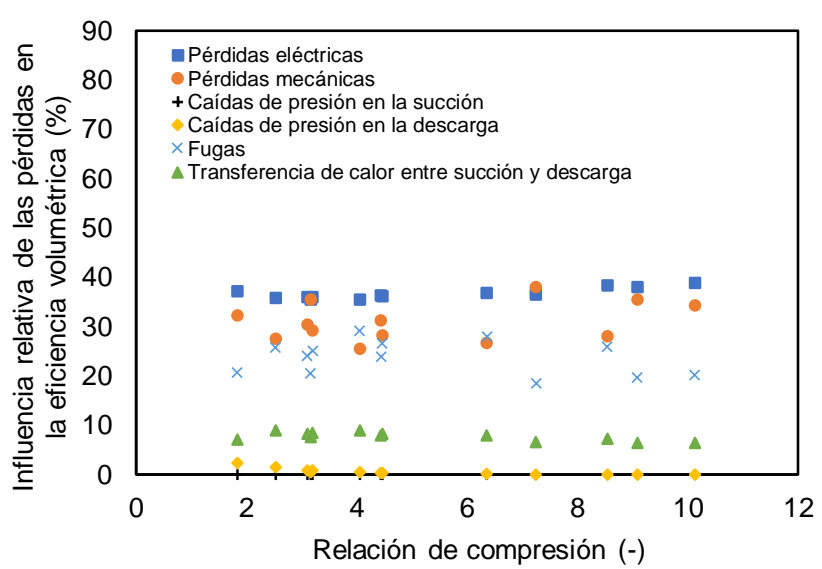

Fig. 5: a) Influencia relativa de las pérdidas consideradas sobre la eficiencia isentrópica del compresor scroll. b) Influencia relativa de las pérdidas consideradas sobre la eficiencia volumétrica del compresor scroll.

Por otro lado, según Navarro et al. (2007a), un compresor alternativo que funciona con R-290 en el punto de trabajo $T_{e}=-7^{\circ} \mathrm{C}, T_{c}=50^{\circ} \mathrm{C}, \mathrm{SH}=10 \mathrm{~K}$, presenta una eficiencia isentrópica de 0.58 y una eficiencia volumétrica de 0.61 . Al comparar estos datos de rendimiento del compresor con los resultados experimentales del compresor scroll operando en las mismas condiciones, el compresor scroll presenta una eficiencia isentrópica de 0.64 , que es un $10 \%$ mayor; y una eficiencia volumétrica de 0.91 , que es un $42 \%$ mayor que la del compresor de pistones. La diferencia en la eficiencia volumétrica se debe a que el compresor de pistones tiene un volumen muerto que degrada su eficiencia volumétrica, la cual a su vez es variable con la velocidad del compresor. A menores velocidades, la eficiencia volumétrica del compresor de pistones puede tener valores más altos. La Tabla 3 muestra la comparación de la influencia relativa de las pérdidas consideradas sobre las eficiencias del compresor para las dos tecnologías de compresores en un punto nominal de operación.

Tabla 3: Influencia relativa de las pérdidas consideradas sobre las eficiencias del compresor para un compresor scroll y un compresor de pistones. Condición de operación $\left(T_{e}=-7^{\circ} \mathrm{C}, T_{C}=50^{\circ} \mathrm{C}, \mathrm{SH}=10 \mathrm{~K}\right)$. Refrigerante R-290.

\begin{tabular}{|c|c|c|c|c|}
\hline \multirow[t]{2}{*}{ Pérdidas } & \multicolumn{2}{|c|}{ Compresor scroll } & \multicolumn{2}{|c|}{$\begin{array}{l}\text { Compresor de pistones } \\
\text { (Navarro et al., 2007a) }\end{array}$} \\
\hline & Inf. Rel. sobre $\eta_{c}(\%)$ & Inf. Rel. sobre $\eta_{v}(\%)$ & Inf. Rel. sobre $\eta_{c}(\%)$ & Inf. Rel. sobre $\eta_{v}(\%)$ \\
\hline Mecánicas & 42 & 32 & 38 & 20 \\
\hline Eléctricas & 40 & 36 & 40 & 26 \\
\hline Pérdidas succión & 1 & 1 & 4 & 14 \\
\hline Pérdidas descarga & 4 & 1 & 2 & 1 \\
\hline Fugas & 10 & 23 & 9 & 6 \\
\hline Transferencia de calor & 3 & 7 & 7 & 15 \\
\hline
\end{tabular}


En cuanto a la eficiencia isentrópica, las pérdidas mecánicas, eléctricas y las fugas presentan una influencia similar en los dos compresores de aproximadamente un $90 \%$; mientras que las pérdidas por caídas de presión en la succión y transferencia de calor entre la descarga y la succión son aproximadamente 7\% más influyentes en el compresor de pistones. Las pérdidas de presión en la descarga son un $2 \%$ más influyentes en el compresor scroll. Por lo tanto, la diferencia más notable con respecto a la eficiencia isentrópica es la influencia de las pérdidas de presión en la succión y en la transferencia de calor entre la succión y la descarga.

En cuanto a la eficiencia volumétrica, las pérdidas mecánicas, eléctricas y las fugas tienen una mayor influencia en el compresor scroll, aproximadamente un 39\%; mientras que las pérdidas de presión en la succión y la trasferencia de calor entre succión y descarga son un $21 \%$ más influyentes en el compresor de pistones. Estas pérdidas son importantes debido a que el compresor de pistones tiene una válvula en la cámara de succión y, además, la diferencia de temperatura entre la succión y descarga es mayor que en el compresor scroll. En cambio, el compresor scroll no tiene válvula en la succión y la diferencia de temperatura entre las diferentes cámaras de compresión que se forman entre las espirales es menor. Se debe tomar en cuenta que los resultados mostrados en el presente estudio, con respecto a las fugas, muestran su influencia sobre la reducción de las eficiencias de los compresores y no los valores absolutos de fugas de refrigerante. Las fugas presentan un alto impacto en la eficiencia volumétrica para el compresor scroll (23\%) en comparación con el compresor alternativo (6\%) como se muestra en la Tabla 3. Sin embargo, el flujo másico de fugas en el compresor scroll con respecto al flujo másico total es del $2.75 \%$, el cual es menor que el flujo másico de fugas en el compresor de pistones que es del $4.80 \%$.

\section{CONCLUSIONES}

De los análisis realizados en el presente estudio, se pueden obtener las siguientes conclusiones sobre la comparación de la influencia relativa de las pérdidas sobre las eficiencias de un compresor scroll y un compresor de pistones: 1) el modelo semi-empírico implementado para compresores scroll considera las principales fuentes de pérdidas energéticas en el proceso de compresión; 2) el modelo se validó experimentalmente ensayando un compresor scroll con R-290 en un banco calorimétrico y reproduce las eficiencias del compresor scroll con un error inferior al $\pm 5 \%$; 3 ) las pérdidas mecánicas, eléctricas y las fugas presentan una influencia en la eficiencia isentrópica similar en los dos compresores de aproximadamente el $90 \%$; 4) la transferencia de calor y las pérdidas de presión en la succión son $7 \%$ más influyentes en la eficiencia isentrópica en el compresor de pistones; 5) respecto a la eficiencia volumétrica, las pérdidas mecánicas, eléctricas y las fugas son aproximadamente 39\% más influyentes en el compresor scroll; 6) la influencia de las pérdidas de presión en la succión y la transferencia de calor son $21 \%$ mayores para el compresor de pistones; y 7 ) el flujo másico de fugas del compresor de pistones es $2.05 \%$ mayor que el del compresor scroll para el punto de trabajo $\mathrm{T}_{e}=-7^{\circ} \mathrm{C}, \mathrm{T}_{\mathrm{c}}=50^{\circ} \mathrm{C}, \mathrm{SH}=10 \mathrm{~K}$.

\section{NOTACIÓN}

$\begin{array}{ll}\text { Símbolos } \\ \mathrm{A}= & \text { área }\left(\mathrm{m}^{2}\right) \\ \mathrm{C}_{\mathrm{p}}= & \text { calor específico }\left(\mathrm{J} \mathrm{kg}^{-1} \mathrm{~K}^{-1}\right) \\ \mathrm{CV}= & \text { válvula de control } \\ \dot{\mathrm{E}}= & \text { potencia eléctrica del compresor }(\mathrm{W}) \\ \mathrm{EEV}= & \text { válvula de expansión electrónica } \\ \dot{\mathrm{E}} \mathrm{L}= & \text { pérdida de energía }(\mathrm{W}) \\ \mathrm{h}= & \text { entalpía específica }\left(\mathrm{J} \mathrm{kg}^{-1}\right) \\ \mathrm{K}_{\mathrm{i}}= & \text { parámetro del compresor } \\ \dot{\mathrm{m}}= & \text { flujo másico }\left(\mathrm{kg} \mathrm{s}^{-1}\right) \\ \mathrm{n}= & \text { velocidad del compresor }(\mathrm{rpm}) \\ \mathrm{P}= & \text { presión }(\mathrm{Pa}) \\ \dot{\mathrm{Q}}= & \text { capacidad }(\mathrm{W}) \\ \mathrm{SH}= & \text { sobrecalentamiento }(\mathrm{K}) \\ \mathrm{T}= & \text { temperatura }\left({ }^{\circ} \mathrm{C}\right) \\ \mathrm{U}= & \text { coeficiente global de transferencia de calor }\left(\mathrm{W} \mathrm{K} \mathrm{K}^{-1} \mathrm{~m}^{-2}\right) \\ \mathrm{V}= & \text { volumen }\left(\mathrm{m}^{3}\right) \\ \dot{\mathrm{V}}= & \text { volumen desplazado }\left(\mathrm{m}^{3} \mathrm{~h}^{-1}\right) \\ \dot{\mathrm{W}}= & \text { trabajo de compresión }(\mathrm{W})\end{array}$

\begin{tabular}{|c|c|}
\hline \multicolumn{2}{|c|}{ Súper/sub índices } \\
\hline $\mathrm{ad}=$ & adaptada \\
\hline$a m b=$ & ambiente \\
\hline$c=$ & condensador \\
\hline des $=$ & descarga \\
\hline$e=$ & evaporador \\
\hline in $=$ & interno \\
\hline $\mathrm{mec}=$ & mecánica \\
\hline$r=$ & relación \\
\hline$s=$ & isentrópico, succión \\
\hline $1=$ & entrada del compresor \\
\hline $8=$ & salida del compresor \\
\hline \multicolumn{2}{|c|}{ Letras Griegas } \\
\hline$\Delta=$ & diferencia \\
\hline$\varepsilon=$ & relación de volúmenes (-) \\
\hline$\rho=$ & densidad $\left(\mathrm{kg} \mathrm{m}^{-3}\right)$ \\
\hline$\eta_{c}=$ & eficiencia isentrópica (-) \\
\hline$\eta_{\mathrm{el}}=$ & eficiencia del motor eléctrico (-) \\
\hline$\eta_{v}=$ & eficiencia volumétrica (-) \\
\hline
\end{tabular}




\section{REFERENCIAS}

Arencibia, K. y Tricio, V., Evaluación Experimental de Compresores Herméticos en Condiciones de Clima Tropical: Eficiencia y Capacidad Frigorífica, http://dx.doi.org/10.4067/S0718-07642014000200012, Inf. Tecnol., 25(2), 103-110 (2014).

ASHRAE Handbook., HVAC Systems and Equipment, American Society of Heating, Refrigerating and Air-Conditioning Engineers, 38.24-38.28, Atlanta, USA (2016).

Bell, I.H., Groll, E.A., Braun, J.E. y Horton, W.T., A Computationally Efficient Hybrid Leakage Model for Positive Displacement Compressors and Expanders, https://doi.org/10.1016/j.jjrefrig.2013.01.005, Int. J. Refrig., 36, 1965-1973 (2013).

Byrne, P., Ghoubali, R. y Miriel, J., Scroll Compressor Modelling for Heat Pumps Using Hydrocarbons as Refrigerants, https://doi.org/10.1016/j.jirefrig.2013.06.003, Int. J. Refrig., 41, 1-13 (2014).

Cuevas, C., Lebrun, J., Lemort, V. y Winandy, E., Characterization of a Scroll Compressor Under Extended Operating Conditions, https://doi.org/10.1016/j.applthermaleng.2009.11.005, Appl. Therm. Eng., 30, 605-615 (2010).

Cuevas, C. y Lebrun, J., Testing and Modelling of a Variable Speed Scroll Compressor, https://doi.org/10.1016/j.applthermaleng.2008.03.016, Appl. Therm. Eng., 29, 469-478 (2009).

Dardenne, L., Fraccari, E., Maggioni, A., Molinaroli, L., Proserpio, L. y Winandy, E., Semi-empirical Modelling of a Variable Speed Scroll Compressor with vapour injection, https://doi.org/10.1016/j.jirefrig.2015.03.004, Int. J. Refrig., 54, 76-87 (2015).

Duprez, M. E., Dumont, E. y Frère, M., Modelling of Reciprocating and Scroll Compressors, https://doi.org/10.1016/j.ijrefrig.2006.11.014, Int. J. Refrig., 30, 873-886 (2007).

Duprez, M. E., Dumont, E. y Frère, M., Modeling of Scroll Compressors-Improvements, https://doi.org/10.1016/j.jirefrig.2009.12.025, Int. J. Refrig., 33, 721-728 (2010).

EN 13771-1., Compressors and Condensing Units for Refrigeration - Performance Testing and Test Methods - Part 1: Refrigerant compressors, European Committee for Standardization, Brussels, Belgium: EN Standard (2017).

EN 378., Refrigerating Systems and Heat pumps - Safety and Environmental Requirements, European Committee for Standardization, Brussels, Belgium: EN Standard (2017).

Giuffrida, A., Modelling the Performance of a Scroll Expander for Small Organic Rankine Cycles when Changing the Working Fluid, https://doi.org/10.1016/j.applthermaleng.2014.06.004, Appl. Therm. Eng., 70, 1040-1049 (2014).

James, N.A., Braun, J.E., Groll, E.A. y Horton, W.T., Semi-empirical Modeling and Analysis of Oil Flooded R410A Scroll Compressors with Liquid Injection for Use in Vapor Compression Systems, https://doi.org/10.1016/j.jirefrig.2015.12.011, Int. J. Refrig., 66, 50-63 (2016).

Klein, S.A. y Alvarado, F.L., EES-Engineering Equation Solver, Academic Professional Version 10.091, F-Chart Software, Madison, WI, USA (2017).

Lemmon, E., Huber, M. y Mc Linden, M., NIST Standard Reference Database 23: Reference Fluid Thermodynamic and Transport Properties-refprop, Version 9.0, Gaithersburg: National Institute of Standards and Technology, Standard Reference Data Program (2010).

Navarro, E., Granryd, E., Urchueguía, J.F. y Corberán, J.M., A Phenomenological Model for Analyzing Reciprocating Compressors, https://doi.org/10.1016/j.jjrefrig.2007.02.006, Int. J. Refrig., 30, 1254-1265 (2007a).

Navarro, E., Urchueguía, J.F., Corberán, J.M. y Granryd, E., Performance Analysis of a Series of Hermetic Reciprocating Compressors Working with R290 (propane) and R407C, https://doi.org/10.1016/j.jirefrig.2007.02.004, Int. J. Refrig., 30, 1244-1253 (2007b).

Pereira, E.L. y Deschamps, C.J., Numerical Analysis and Correlations for Radial and Tangential Leakage of Gas in Scroll Compressors, https://doi.org/10.1016/j.ijrefrig.2019.11.002, Int. J. Refrig., 110, 239-247 (2020).

Press, W.H., Teukolsky, S.A., Vetterling, W.T. y Flannery, B.P., Numerical Recipes: The Art of Scientific Computing, 3rd Ed. ed. New York: Cambridge University Press (2007).

Roskosch, D., Venzik, V. y Atakan, B., Thermodynamic Model for Reciprocating Compressors with the Focus on Fluid Dependent Efficiencies, https://doi.org/10.1016/j.ijrefrig.2017.08.011, Int. J. Ref., 84, 104-116 (2017).

Tello-Oquendo, F.M., Navarro-Peris, E., Barceló-Ruescas, F. y Gonzálvez-Maciá, J., Semi-empirical Model of Scroll Compressor and its Extention to Describe Vapor-Injection Compressors. Model Description and Experimental Validation, https://doi.org/10.1016/j.ijrefrig.2019.06.031, Int. J. Refrig., 106, 308-326 (2018).

Tuhovcak, J., Hejcik, J. y Jicha, M., Comparison of Heat Transfer Models for Reciprocating Compressor, https://doi.org/10.1016/j.applthermaleng.2016.04.120, Appl. Therm. Eng., 103, 607-615 (2016).

Winandy, E., Saavedra, C. y Lebrun, J., Experimental Analysis and Simplified Modelling of a Hermetic Scroll Refrigeration Compressor, https://doi.org/10.1016/S1359-4311(01)00083-7, Appl. Therm. Eng., 22, 107-120 (2002). 